\title{
Light recycling beam steering on a DMD lidar
}

Guanghao Chen, Brandon Hellman, Joshua Rodriguez, Braden Smith, Adley Gin, et al.

Guanghao Chen, Brandon Hellman, Joshua Rodriguez, Braden Smith, Adley Gin, Yuzuru Takashima, "Light recycling beam steering on a DMD lidar," Proc. SPIE 10757, Optical Data Storage 2018: Industrial Optical Devices and Systems, 107570G (14 September 2018); doi: 10.1117/12.2323876

SPIE Event: SPIE Optical Engineering + Applications, 2018, San Diego, California, United States 


\title{
Light Recycling Beam Steering on a DMD Lidar
}

\author{
Guanghao Chen ${ }^{\mathrm{a}}$, Brandon Hellman ${ }^{\mathrm{a}}$, Joshua Rodriguez ${ }^{\mathrm{a}}$, Braden Smith ${ }^{\mathrm{b}}$, Adley Ginª, and Yuzuru \\ Takashima*a $^{* a}$ \\ ${ }^{a}$ University of Arizona, College of Optical Science, 1630 E. University Blvd., Tucson, AZ USA \\ 85719; 'bandia National Laboratory, 1515 Eubank Blvd. SE, Albuquerque, NM, USA 87123
}

\begin{abstract}
Spatial light modulators (SLMs) that operate in a phase modulation mode enable beam steering with higher diffraction efficiency compared to amplitude modulation mode, thus potentially be used for an efficient beam steering with no moving part. Currently, Twisted Nematic phase SLMs are widely adopted for phase modulation. However, their refresh rate is typically in the range below kilohertz. Recently, a new method for binary and spatial phase modulation using Digital Micromirror Device (DMD) was proposed by a research group in Germany. In the method, complemental self-images of DMD, corresponding to on- and off-pixels, are formed by two auxiliary optics while adding a pi phase shift between two images. The optics function as recycling of light in a coherent manner. The method enables over kilohertz refresh rate and higher diffraction efficiency in binary phase modulation mode to conventional amplitude binary modulation.

As alternatives to the binary phase modulation, we propose and experimentally evaluated high-speed beam steering by DMD based on light recycling. In our experiment, with binary phase modulation mode, system output efficiency reaches $8 \%$. It can be doubled to $16 \%$ with light recycling method. Efficiency is still low compared to the reported value of $27 \%$ without light recycling. To further increase beam efficiency, system loss was analysed.
\end{abstract}

\section{INTRODUCTION}

In recent years, lidar (light detection and ranging) has been widely adopted in autonomous vehicles and robot visions. In a lidar system, beam steering is necessary for signal emission and detection. Laser beam steering techniques have been applied in laser printers, optical communication systems, microscopes, laser processing, etc. However, conventional techniques encountered difficulties meeting the requirements on vehicle and robot platforms, such as immense vibrations, high-speed detection and processing, low power consumption, compact form factors, and high resolution. Nowadays, popular steering methods on these platforms include mechanical steering, MEMS (microelectromechanical system) steering, and phased array steering.

While mechanical steering, a gimbal system for example, is the most mature and popular solution, it is also the most expensive when compared to other methods. The size and cost of this type of devices greatly limit the overall performance, such as speed and resolution. Very few can be installed on a single platform, let alone issues such as vibrations, durability, lack of random access, and power consumptions.

In contrast, MEMS and phased array techniques are more flexible for such applications. These techniques are compatible with current semiconductor fabrication process, which allows mass and low-cost production. The low power consumption and small form factors greatly increase manufacturers' desires to install multiple devices on a single platform, which in return enhances robustness and overall performance of the system. Among these methods, nanophotonic phased array (NPA) is still under development. Difficulties arise when scientists try to generate enough power for long range detection while maintain a wide steering angle ${ }^{[1]}$, let along the limited selectable wavelengths due to the transparency window of silicon $^{[2]}$. Albeit these issues exist, NPA steering is still promising for applications such as display, imaging, and communications for the advantages it bears.

Meanwhile, researchers also investigate MEMS mirror steering technologies. MEMS mirrors flip mechanically at a high frequency. Same as NPAs, MEMS mirrors can scan a large angle rapidly, while maintain high output efficiencies over a

*ytakashima@optics.arizona.edu 
broad range of wavelengths and thus can emit more power for long range detection. Besides, some devices can function normally at extreme conditions such as low/high temperature, making them a feasible beam steering solution for compact Lidar systems $^{[3]}$.

In this paper, we demonstrate our efforts on improving the angular resolution of the system proposed in [4]. In that paper, Smith et al. demonstrated a single-chip DMD beam steering method. In the design, the mirror array functions like a blazed grating with variable blazed angles. By precisely synchronizing the pulsed laser and the DMD frame, one can equivalently freeze the mirror array at a specific angle between $-12^{\circ}$ and $12^{\circ}$. As shown in Eq.(1), when blazed angle changes, phase retardance induced changes as well ${ }^{[5]}$. Therefore, output power in one order shifts to the adjacent order and beam steering is achieved. Although many diffraction orders can be observed, as a blazed grating, most of the energy will fall on the blazed order and diffraction efficiency is as high as $90 \%$.

$$
\eta_{q}=\operatorname{sinc}^{2}\left(\frac{q}{2^{N}}\right) \frac{\operatorname{sinc}^{2}\left(q-\frac{\phi_{0}}{2 \pi}\right)}{\operatorname{sinc}^{2}\left(\frac{q-\frac{\phi_{0}}{2 \pi}}{2^{N}}\right)}
$$

This method combines the advantages of DMD technology we mentioned previously and can output much higher power than NPAs. Compared to conventional mechanical beam steering devices, DMD scanning devices are compact and power efficient. Mass production at low cost is also achievable. A problem that we found is, although a DMD scanning device can sweep beams over a large angle, the angular resolution is constrained by the DMD pixel pitch. To address this problem, we introduce a holographic beam steering method that can further increase the angular resolution of the DMD beam scanning device.

In section 2, we briefly introduce the principles of holography beam steering and the computer-generated holograms (CGH). Light recycling method is also proposed in this section. In section 3, experiment details and experimental results are shown and analysed. Particularly, in section 4, we analysed the system loss. Conclusions of the paper are made in section 5 .

\section{INTRODUCTION TO HOLOGRAPHY BEAM STEERING, TWIN IMAGE REMOVAL AND LIGHT RECYCLING}

The idea of increasing angular resolution of the blazed grating design is realized by shifting the angle of the incident beam. Alternative design was also proposed by Rodriguez et al. in which multiple laser sources are placed at slightly different incident angles and each create a set of diffraction orders with an equal angle shift ${ }^{[6]}$. However, as angular resolution increases, one must tweak the optical design to accommodate more lasers and fire the laser pulses in a precise manner to achieve accurate detection. By contrast, holographic beam steering in our current design requires only two DMDs at most and achieved an angular resolution 48 times finer than blazed grating coarse scanning.

Essentially, holography beam steering is achieved by wavefront reconstruction of computer-generated holograms (CGH). A hologram is illuminated by an incident beam (probe beam). A reconstructed wavefront propagates towards the desired direction and thus beam steering is achieved. To generate the holograms, we used a virtual hologram recording process in which we defined the reference wavefront and the signal wavefront in a MATLAB program. The DMD is considered as a recording medium and is defined in the interfering region where reference beam and signal beam intersect. All parameters must match the physical configurations of the DMD used. In our research, a DLP3000 DMD (Texas Instruments) is used for hologram display. The resolution is $608 \times 684$ with a pixel pitch of $7.6 \mu \mathrm{m}$. Maximum FPS is $4000 \mathrm{~Hz}$ with mirrors flipped to $\pm 12^{\circ[7]}$. 


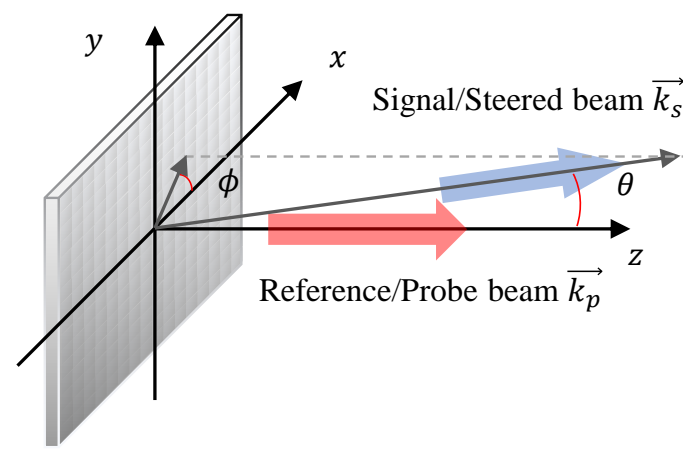

(a)

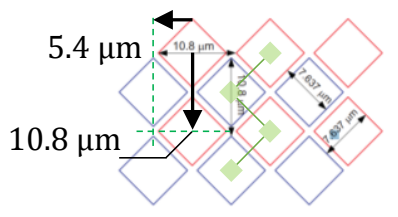

(b)
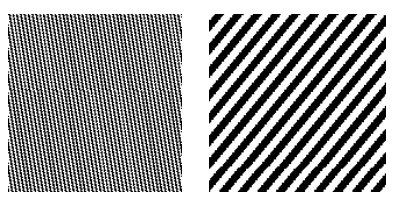

(c)

Figure 1 DMD micromirror layout and virtual recording setup.

(a) is a coordinate system established on the DMD in our virtual recording setup; (b) is adapted from Fig.6 in [7]. In this micromirror layout, the row pitch is half of the column pitch. The former is $5.4 \mu \mathrm{m}$, while the latter is $10.8 \mu \mathrm{m}$. The green "zig-zag" pixel chain denotes the micromirrors that used to display one image pixel column; (c) are the holograms recorded. The denser the fringes are, the larger the steer angle is.

In our program, a coordinate system is established with its origin located at the centre of the DMD surface, shown as Figure 1 (a). The k-vectors of the signal beam and the reference beam are defined on this coordinate, shown as the following. On this coordinate, the k-vectors of the reference beam and the signal beam are defined as

$$
\begin{gathered}
\overrightarrow{k_{p}}=k_{p} \hat{z} \\
\overrightarrow{k_{s}}=k_{s}(\sin \theta \cos \phi \hat{x}+\sin \theta \sin \phi \hat{y}+\cos \theta \hat{z})
\end{gathered}
$$

Two plane waves are thus defined with the two k-vectors. So long as the spatial coordinates of all the pixels are defined, the interferogram would be sampled by these pixels. The maximum steer angle is limited by Nyquist-Shannon sampling theorem. By contrast, it takes at least two pixels to represent a varying signal in holography scanning, while in coarse scanning, each micromirror spans a grating period. Therefore, the maximum spatial frequency is

$$
k_{\max }=\frac{1}{2 \Delta x}
$$

By diffraction theory ${ }^{[5]}$, it is known that

$$
k_{\max }=\frac{x_{\max }}{\lambda z}=\frac{\tan \theta_{\max }}{\lambda} \approx \frac{\theta_{\max }}{\lambda}, \quad \theta_{\max } \text { is small. }
$$

where $\Delta x$ denotes the pixel pitch and $\Delta \mathrm{x}=7.637 \mu \mathrm{m}$ on this device. $k_{\max }$ is equal to $6.547 \times 10^{4} \mathrm{~m}^{-1}$. Hence, when illuminated with $532 \mathrm{~nm}$ coherent light, $\theta_{\max }$ approximates $\pm 2^{\circ}$, meaning that in our virtual recording process, the angle between the signal beam and the reference beam should be less than $\theta_{\max }$ to prevent aliasing. This angle is half of the angular spacing in coarse scanning mode and is confirmed experimentally.

It is worth mentioning that the micromirrors are tiled with each square mirror rotated $45^{\circ}$ (diamond shape). Therefore, a column of image frame pixels is displayed by two columns, one blue column and one red column, of micromirrors in a "zig-zag" sequence as labelled by the green line on Figure 1 (b). In such layout, the row pitch is half of the column pitch. The former is $5.4 \mu \mathrm{m}$ and the latter is $10.8 \mu \mathrm{m}$. Patterns on (c) are the holograms recorded with this virtual process.

A test was carried out by displaying a binary amplitude hologram on the DMD. Diffraction far-field pattern is shown in Figure 2. Multiple diffraction images were formed, including the $0^{\text {th }}$ order (DC image), a real image of $0^{\text {th }}$ order, a conjugate image of $0^{\text {th }}$ order, and images from the adjacent orders. 


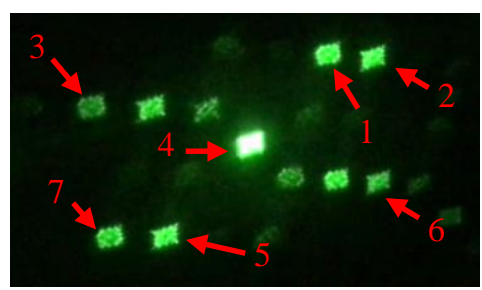

Figure 2 Test result of DMD amplitude modulation

Arrow 1 indicates the desired image in the $0^{\text {th }}$ order; arrow 2 and 3 indicate the image in adjacent order; arrow 4 indicates the DC component. Arrow 5-7 indicate the conjugates (twin images) of the images pointed by arrow 1-3.

Figure 2 shows the far-field patterns. The desired steered bema is pointed by arrow 1 and was moving from the left of the field to the right. The other patterns are the DC image and conjugate images of the field. Aliasing occurred as we can see the pattern of the adjacent orders locates closer to the DC image. They can be easily removed by limiting the steer angles. Since the reconstructed image is a plane wave, the twin image is therefore a plane wave and can be easily seen in the experiment.

To eliminate the unwanted components, a single-sideband spatial filtering approach is applied ${ }^{[8]}$. Instead of letting the light converge in infinity, a 4- $f$ system was inserted in our system to spatially filter some images. Since all the components are plane waves at different angles, they are focused to different locations on the intermediate image. A single-sideband filter is then used to select the desired pattern.

Another issue in holographic beam steering is a low diffraction efficiency due to amplitude based modulation. The holograms displayed on the DMD are effectively Ronchi gratings and theoretically, the $0^{\text {th }}$ order occupies the highest diffraction efficiency, $25 \%$, while the desired pattern and its conjugate share $20.2 \%$ of the incident light. However, in practise, the efficiency is much lower than $10.1 \%$ because of the DMD fill factor is less than 1, shown as Figure 3 (a). Mathematically, the aperture function can be expressed as

$$
u_{\text {aperture }}(x, y)=\operatorname{comb}\left(\frac{x}{L}, \frac{y}{L}\right) \circledast \operatorname{rect}\left(\frac{x}{d}, \frac{y}{d}\right) \cdot u_{\text {hologram }}(x, y) \cdot C_{1}, \quad d \leq L
$$

All the constants are absorbed into $C_{1}$. The fill factor is defined as

$$
\text { Fill factor }=\left(\frac{d}{L}\right)^{2}
$$

It equals to $92 \%$ on this model ${ }^{[7]}$. The far field intensity distribution is

$$
\begin{aligned}
& I(\xi, \eta)=\left|F_{2}\left\{u_{\text {aperture }}\right\}\right|^{2} \\
& =\left|\operatorname{comb}(L \xi, L \eta) \cdot \operatorname{sinc}(d \xi, d \eta) \circledast F_{2}\left\{u_{\text {hologram }}\right\} \cdot C_{2}\right|_{\xi=\frac{x}{\lambda z}, \eta=\frac{y}{\lambda z}}^{2}
\end{aligned}
$$

All the constants are absorbed into $C_{2} . F_{2}\left\{u_{\text {hologram }}\right\}$ is the 2D Fourier transform of the hologram, which yields the reconstruction image. By simply looking at the first two terms in Eq. (7), we can see that, if $d=L$, fill factor equals 1 and

$$
\iint_{\xi \neq 0, \eta \neq 0}|\operatorname{comb}(L \xi, L \eta) \cdot \sin c(d \xi, d \eta)|^{2} d \xi d \eta=0
$$

meaning that all the energy is in the 0th order. Otherwise, this integral is larger than 0 , meaning that the energy leaks to other diffraction orders, which occurs in our experiment and substantially lowers the diffraction efficiency of the 0th order. 


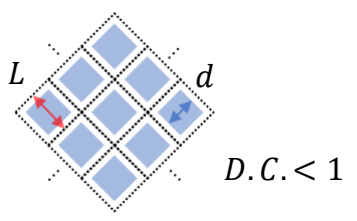

(a)

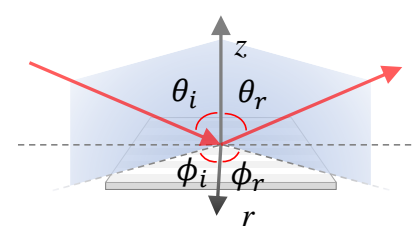

(b)

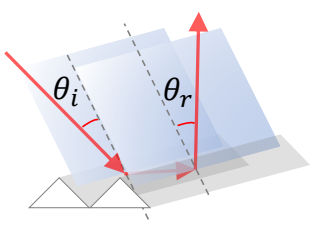

Figure 3 A 1D retroreflector.

(a) is a drawing of a DMD array. The fill factor is less than 1. A micromirror doesn't span all the area defined by the pixel pitch. (b) shows the overview of a 1D retroreflector and its detailed drawing. The incident angles $\theta_{i}$ and reflective angle $\theta_{r}$ are still in accord with Snell's law, but the incident plane and reflection plane are in symmetry of $r$-axis.

In our research, we proposed to recycle the twin image to increase the output efficiency. An explanatory drawing is shown in Figure 3 (b). Since the wavefronts of both the steered beam and its twin image are plane waves, we can fold the twin image to the same direction as the steered beam with a 1D retroreflector to double the output efficiency. However, the overall efficiency is still greatly limited by the nature of amplitude modulation mode. In the next section, we will introduce our efforts on using a DMD as a phase modulator.

\section{STEERING BEAMS WITH BINARY PHASE GRATINGS ON A DMD}

Ideally, a binary amplitude Ronchi grating can diffract $10.1 \%$ of the incident power to $\pm 1^{\text {st }}$ orders $(20.2 \%$ in total), while $25 \%$ of the power remains in the $0^{\text {th }}$ order. $50 \%$ of the total power is absorbed by the grating. The low diffraction efficiency makes the amplitude based holographic scanning less desirable. A solution to this problem is to use phase holograms. Theoretically, a binary phase grating can eliminate the $0^{\text {th }}$ order and direct $40.5 \%$ (81\% in total) of the incident power into $\pm 1^{\text {st }}$ orders equally. The grating doesn't absorb any power. Liquid crystals are very suitable for phase modulation. The fastest ferroelectric LC binary phase SLMs can operate at several kilohertz, yet, they are expensive and the modulation speed is limited within kilohertz range, while the current fastest DMD can operate at $22 \mathrm{kHz}$. Several efforts have been made to convert a DMD into a phase modulator ${ }^{[5-8]}$, however, these methods achieve phase modulation at the cost of diffraction efficiency. Among the efforts, Hoffmann et al. proposed an interesting system design in [13] and successfully achieved real binary phase modulation on a single DMD. $4 \mathrm{kHz}$ binary phase modulation with $27 \%$ maximum diffraction efficiency is reported in their research. Hence, we are encouraged to implement a similar system in our research in beam steering with holograms.

In the symmetric system proposed, the beams deflected from the micromirrors are collected by two 4- $f$ systems placed at $24^{\circ}$ to the normal of the DMD. A schematic adapted from the original design is shown in Figure 4 (a). Some modifications have been made. If a binary amplitude image is displayed on the DMD, the incoming light will be deflected to the left arm, if it hits an "on" pixel at $12^{\circ}$; or it will be deflected to the right arm, if it hits an "off" pixel at $-12^{\circ}$. The mirror in the left arm is attached to a PZT and is precisely controlled such that it introduces $\pi$ phase retardance between both arms. The phase modulated light is then imaged to the other half of the DMD. 


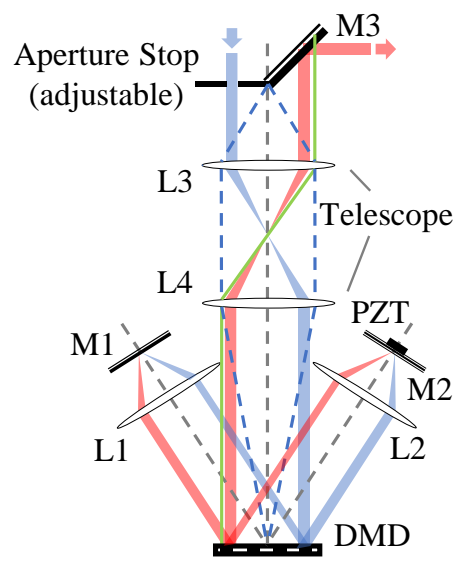

(a)

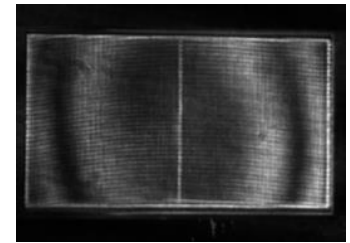

(b)

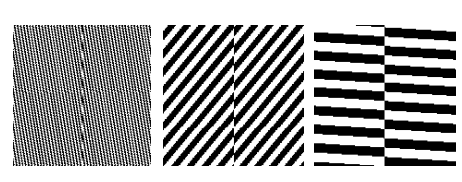

(c)

Figure 4. DMD binary phase modulator, a Moiré pattern, and binary phase holograms.

(a) is a schematic of the system used in our research. Some modifications have been made to the original design in ${ }^{[13]}$. (b) shows the Moiré fringes on the DMD surface in alignment mode. (c) shows examples of DMD beam steering binary phase holograms. The bright and dark regions are of $\pi$ phase difference. These holograms are point symmetric due to the symmetric system layout.

In our setup, M1 and M2 are two first surface mirrors. M2 is mounted on a piezoelectric transducer (PZT, F4010885, Burleigh Instruments). L1 and L2 are two identical lenses $(f=122 \mathrm{~mm}, \mathrm{~F} / 4.8)$. L3 and L4 together is an inverse telescope. The adjustable aperture stop is imaged by the telescope onto the DMD to counter diffraction effect. After beams exit both arms, they return to the DMD. The field on the DMD is then imaged by the telescope onto the $45^{\circ}$ pickoff mirror and exit the system. In our current experiment, we inserted a 50/50 beam splitter between L4 and the DMD at $45^{\circ}$ to monitor the DMD surface and the size of the aperture precisely with a camera (sensor: DCC1545M, Thorlabs; lens: $f=$ $35 \mathrm{~mm}$, Nikon). This factor is taken into calculation in our loss analysis in the next section.

The challenge of building this system is aligning the $4-f$ systems. The light coming from a micromirror on the DMD must be sent to the diagonal micromirrors without magnification error. Misalignment will lead to wrong modulation and low power efficiency. In our experiment, we used Moiré fringes to aid the alignment process. In an ideal imaging condition where no aberration occurs, defocus will cause image magnification and thus the Moiré patterns will be radially distributed; while image translation will cause Moiré fringe along one direction.

shows the best alignment result we have so far. The Moiré pattern cannot be eliminated due to aberration. Distortions come from the uneven flatness of the glass window and the micromirror array, and/or aberrations in L1, L2, and mirrors.

Examples of binary phase holograms are shown in Figure 4 (c). Considering that the image from the left half of the DMD is flipped horizontally and vertically, and imaged to the right half of the DMD, the combined image is point symmetric. Therefore, we see a sharp cut in the holograms.

\section{SYSTEM LOSS ANALYSIS}

To confirm that the system was operating in phase modulation mode, we measured the diffraction efficiencies of different diffraction orders. A setup for measurement is built as Figure 5 (a). A sensor array (DCC1545M, Thorlabs) was used to capture the $0^{\text {th }}$ and $\pm 1^{\text {st }}$ orders. They are, in indeed, the interferograms of two-beam interference. The unevenness shows that the wavefront curvatures of the beams are different. By tuning the PZT controller (Burleigh Instruments), we can change the phase difference. In open-loop control mode, the phase difference is not locked and shifts the intensities of the orders rapidly. 


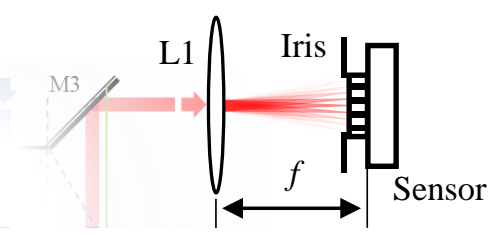

(a)

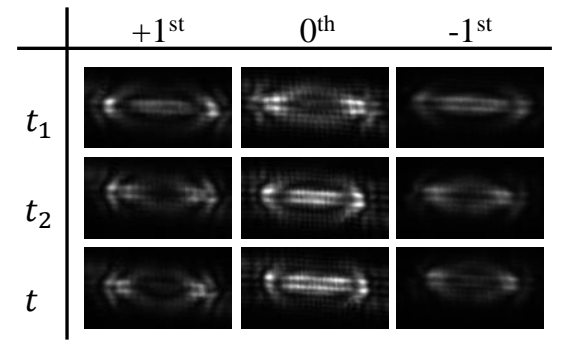

(b)

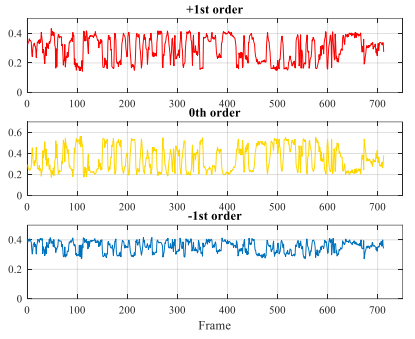

(c)

Figure 5. Setup for diffraction efficiency measurement, measurement results, and power ratio time sequences. In (a) the sensor (imaging or power detection) is placed at the focal length of L1. An iris is used to select one or more diffraction orders. (b) shows the intensity distributions of different diffraction orders at different time. (c) shows an example of the power ratio sequences analysed by the program.

Figure 5 (b) shows the intensities distributions of the three orders at different time. A MATLAB program is used to analyse the image sequence of the intensities and calculate the power ratio of each order with different window sizes based on the equation below. An example of power ratio sequences is given in Figure 5 (c).

$$
\text { Power ratio of } \mathrm{m}^{\text {th }} \text { order }=\frac{\text { Power of } \mathrm{m}^{\text {th }} \text { order }}{\sum_{\mathrm{m}=-1}^{+1} \text { Power of } \mathrm{m}^{\text {th }} \text { order }}
$$

Based on this equation, in amplitude modulation, $+1^{\text {st }}$ order should take up around $22 \%$ of the power among the them, while in the phase modulation mode we shown above, the maximum recorded power ratio of the $+1^{\text {st }}$ order is higher than $36 \%$. It reached $41.7 \%$ when a smaller aperture was used, yet, it is not completely phase modulation, since the ideal ratio is $50 \%$ in real phase modulation. The total measured output efficiency of the system is $9.6 \%$ and it is measured with the beam splitter inserted in the path. Without the $50 / 50$ beam splitter, the efficiency is doubled to $19.2 \%$.

After measuring the power ratios of diffraction orders, we measured the efficiencies of different parts in the system. Measurement results are shown in Table 1. The total estimated efficiency is close to the total measured. With this analysis, we have a better knowledge of improving system performance.

Table 1. System Efficiency Break-down (50/50 beam splitter inserted)

\begin{tabular}{ll}
\hline Scenario & Efficiency \\
\hline Lenses only receive part of the light from the DMD & $45.09 \%$ \\
Transmission of the lenses & $72.14 \%$ \\
Mirrors only capture part of the light from the lenses & $88.76 \%$ \\
Lenses only capture part of the wavefront from the mirrors & $66.98 \%$ \\
Beam splitter & $50 \%$ \\
Total estimated & $8.32 \%$ \\
Total measured & $9.6 \%$ \\
\hline
\end{tabular}

In summary, using the diffraction efficiency mentioned in Table 1, the expected efficiency of the steered beam is around $4 \%$ (8\% without beam splitter), which is much lower than $27 \%$ mention in [13]. It can be further improved from several aspects. First, the DMD used in their research has a larger pixel pitch, $13.68 \mu \mathrm{m}$, which is easier for system alignment. Second, for lens L1 and L2 in Figure 4, the F-number is f/3, while it is f/4.8 in our system and the light from the DMD and from the mirrors are not fully captured. Third, M1 and M2 are not large enough to capture all the spatial frequencies. Other 
factors include anti-reflection coatings, DMD flatness, and vibrations. At last, we can double the efficiency to $16 \%$ with light recycling method.

\section{CONCLUSIONS}

We introduced our research in beam steering with DMD phase holograms. A MATLAB program is used to simulate the holography recording process and generate the required holograms. Maximum steer angle approximates $\pm 2^{\circ}$. A singlesideband method proposed in [8] is implemented in our system to remove the unwanted diffraction patterns. We also proposed light recycling method that utilizes the twin image to double the output efficiency. A DMD binary phase modulator design from [13] is used. Total measured output efficiency in our system is around 8\%. With light recycling method implemented, the efficiency is expected to be $16 \%$.

\section{REFERENCES}

[1] D. Hutchison, J. Sun, J. Doylend, R. Kumar, J. H.- Optica, and undefined 2016, "High-resolution aliasing-free optical beam steering," osapublishing.org.

[2] C. V. Poulton et al., "Coherent solid-state LIDAR with silicon photonic optical phased arrays," Opt. Lett., vol. 42, no. 20 , p. 4091 , Oct. 2017.

[3] "DLP3030-Q1 0.3 WVGA S450 DMD.” Texas Instruments.

[4] B. Smith, B. Hellman, A. Gin, A. Espinoza, and Y. Takashima, "Single chip lidar with discrete beam steering by digital micromirror device," Opt. Express, vol. 25, no. 13, p. 14732, Jun. 2017.

[5] J. W. Goodman, "Introduction to Fourier Optics 2ed," Book, 1968.

[6] J. Rodriguez, B. Smith, B. Hellman, A. Gin, Y. Takashima, and A. J. Espinoza, "Multi-beam and single-chip LIDAR with discrete beam-steering by digital micromirror device," Phys. Simul. Optoelectron. Devices XXVI, vol. 10526, no. February, p. 29, Feb. 2018.

[7] “DLP3000 DLP ${ }^{\circledR} 0.3$ WVGA Series 220 DMD.” Texas Instruments.

[8] Y. Takaki and Y. Tanemoto, "Band-limited zone plates for single-sideband holography.," Appl. Opt., vol. 48, no. 34, pp. H64-70, 2009.

[9] W. H. Lee, "III Computer-Generated Holograms: Techniques and Applications," Prog. Opt., vol. 16, no. C, pp. 119-232, Jan. 1978.

[10] I. M. Vellekoop, M. Cui, and C. Yang, "Digital optical phase conjugation of fluorescence in turbid tissue," Appl. Phys. Lett., vol. 101, no. 8, p. 081108, Aug. 2012.

[11] S. A. Goorden, J. Bertolotti, and A. P. Mosk, "Superpixel-based spatial amplitude and phase modulation using a digital micromirror device," Opt. Express, vol. 22, no. 15, p. 17999, Jul. 2014.

[12] D. B. Conkey, A. M. Caravaca-Aguirre, and R. Piestun, "High-speed scattering medium characterization with application to focusing light through turbid media," Opt. Express, vol. 20, no. 2, p. 1733, Jan. 2012.

[13] M. Hoffmann, I. N. Papadopoulos, and B. Judkewitz, "Kilohertz binary phase modulator for pulsed laser sources using a digital micromirror device," Oct. 2017. 